\title{
Écoulement turbulent entre un disque tournant et un disque fixe, influence des phénomènes d'entrée liés à la valeur finie des rayons
}

\author{
ABDELKader NouredDine ${ }^{a}$ \\ Laboratoire de Mécanique Appliquée, Faculté de Génie Mécanique, U.S.T.O-MB.Oran, BP 1505 Oran El M’Naouer, Algérie
}

Reçu le 15 décembre 2008, accepté le 10 septembre 2009

\begin{abstract}
Résumé - Cet article illustre la mise en évidence des phénomènes d'entrée liés à la valeur finie des rayons de deux disques, l'un est tournant et l'autre est fixe. L'analyse de l'écoulement est faite à partir d'une configuration des lignes de courant pariétales induites au voisinage d'un disque tournant en utilisant la technique de visualisation de l'écoulement pariétal par dépôt électrolytique qui a donné de bons résultats dans plusieurs cas de solides en rotation. Cette méthode expérimentale a permis de montrer les différents phénomènes caractérisant ce type d'écoulement tels que les effets de bord et de vérifier les résultats théoriques concernant le champ des vitesses obtenues grâce à un modèle mathématique basé sur une méthode itérative à tir corrigé. L'écoulement est de type Batchelor : deux couches limites développées sur chaque disque, séparées par un noyau de fluide tournant à $0,3 \omega$ ( $\omega$ étant la vitesse de rotation du disque tournant).
\end{abstract}

Mots clés : Visualisation / ligne de courant pariétal / disque fixe / disque tournant / effets d'entrée

Abstract - Turbulent flow between rotating disc and fixed disc, influence of edge effects with the fixed radius values. This paper illustrates the influence of the finite radius of two disks on the inlet phenomena. The configuration of the streamlines of the flow in the vicinity of a rotating disc is considered. Good results are obtained using the electrolytic deposit to visualize the parietal flow in the case of rotating solids. This experimental technique enabled to show different phenomena characterizing the flow types such as the edge effects and to validate the theoretical results concerning the velocity field obtained by a mathematical model based on a corrected shooting iterative method. The flow is of Batchelor type: two developed boundary layers on each disc, separated by a fluid core rotating with $0.3 \omega$ ( $\omega$ being the disc rotating velocity) were present.

Key words: Visualization / surface streamline / fixed disc / rotating disc / edge effects

\section{Introduction}

Les écoulements au-dessus d'un disque unique ou entre deux disques coaxiaux ont fait l'objet d'un intérêt constant de la part de la communauté scientifique depuis les travaux de Von Karman [1] qui étudia l'écoulement laminaire au-dessus d'un disque tournant de rayon infini dans un fluide initialement au repos. On rencontre ce type d'écoulements dans les domaines industriels tels que la thermique (échangeurs de chaleur), le génie chimique (cellule d'électrolyse), le génie mécanique (butées et paliers hydrostatiques, pompes, ventilateurs). La plupart de ces études théoriques menées par Cochran [2], Bodewat [3], Batchelor [4] ou Rogers et al. [5] décrivant ce problème

\footnotetext{
a Auteur pour correspondance : redkanour@yahoo.fr
}

suppose la dimension radiale des deux disques infinie alors qu'en pratique ils ont toujours un rayon fini.

La technique de visualisation de l'écoulement pariétal par dépôt électrochimique a donné de bons résultats pour différents types d'écoulements qui ont été étudiés par Daguenet et al. [6], Tuhault [7] et Laghouiter et al. [8]. Cette technique est basée sur le principe du transfert de masse vers une surface de réaction de l'électrode sur laquelle on veut visualiser l'écoulement du fluide étudié. Cette méthode de visualisation nous a permis de mettre en évidence les différents éléments caractérisant un écoulement entre un disque tournant et un disque fixe.

Le but de ce travail est de préciser l'étendue de l'influence des phénomènes d'entrée liés à la valeur finie des rayons à partir de la configuration des lignes de courant pariétales. 


\section{Nomenclature}

\begin{tabular}{|ll|}
\hline$\rho:$ Masse volumique & {$\left[\mathrm{k} \cdot \mathrm{m}^{-3}\right]$} \\
$\mu:$ Viscosité dynamique & {$[\mathrm{Pl}]$} \\
$v:$ Viscosité cinématique & {$\left[\mathrm{m}^{2} \cdot \mathrm{s}^{-1}\right]$} \\
$e=e^{*} / r_{o}^{*}:$ Épaisseur réduite adimensionnelle du jeu entre les deux disques & {$[-]$} \\
$r=r^{*} / r_{o}^{*}:$ Rayon réduit adimensionnel des disques & {$[-]$} \\
$r, \theta, z:$ Coordonnées cylindriques adimensionnelles & {$[-]$} \\
$u, v, w:$ Composantes adimensionnelles de la vitesse $v$ & \\
$x, y, z:$ Coordonnées cartésiennes adimensionnelles & \\
$\alpha_{\mathrm{F}}:$ Angle théorique entre la ligne de courant et le rayon vecteur du disque fixe & {$[-]$} \\
$\alpha_{\mathrm{FP}}:$ Angle expérimental entre la ligne de courant et le rayon vecteur du disque fixe & {$[-]$} \\
$\alpha_{\mathrm{T}}:$ Angle théorique entre la ligne de courant et le rayon vecteur du disque tournant & {$[-]$} \\
$\alpha \mathrm{TP}:$ Angle expérimental entre la ligne de courant et le rayon vecteur du disque tournant & {$[-]$} \\
$\omega:$ Vitesse angulaire du disque tournant & {$\left[\mathrm{s}^{-1}\right]$} \\
$R e=\omega e^{* 2} / v:$ Nombre de Reynolds de rotation & {$[-]$} \\
\hline
\end{tabular}

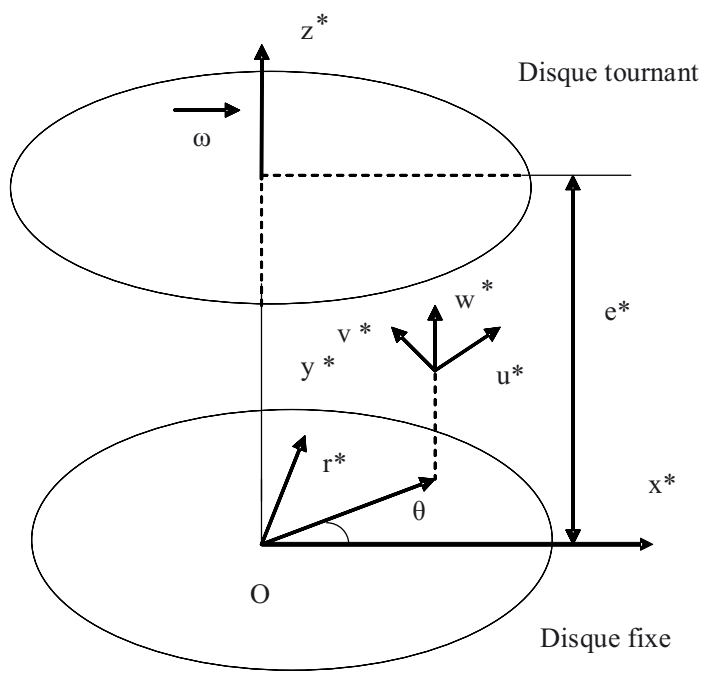

Fig. 1. Position du problème.

\section{Position du problème}

On considère l'écoulement d'un fluide visqueux incompressible entre deux disques coaxiaux, parallèles dont l'un est en rotation. On suppose que le régime est laminaire et permanent. On désigne respectivement par $\rho$ et $\nu$ la masse volumique et la viscosité cinématique du fluide.

Le disque tournant est situé à la cote $z^{*}=e^{*}$ et tourne autour de l'axe $O z^{*}$ avec une vitesse angulaire constante $\omega$. Le disque fixe est situé à la cote $z^{*}=0$ comme le montre la figure 1. Compte tenu des hypothèses, les équations de Navier-Stokes et l'équation de continuité s'écrivent :

$$
\left.\begin{array}{l}
\overline{\overline{\operatorname{grad}}} \vec{V}^{*} \cdot \vec{V}^{*}=-\frac{1}{\rho}\left(\operatorname{grad} p^{*}\right)+v \Delta \vec{V}^{*} \\
\operatorname{div} \vec{V}^{*}=0
\end{array}\right\}
$$

$p^{*}\left(r^{*}, \theta, z^{*}\right)$ désigne la pression motrice au point $\mathrm{M}$ $\left(r^{*}, \theta, z^{*}\right)$.
Les conditions aux limites sont :

$$
\left.\begin{array}{l}
\vec{V}^{*}\left(r^{*}, \theta, 0\right)=\overrightarrow{0} \\
\vec{V}^{*}\left(r^{*}, \theta, e^{*}\right)=\omega r^{*} \vec{j}
\end{array}\right\}
$$

Les données dimensionnelles du problème sont au nombre de $4: e, \omega, \mu, \rho$. Afin d'obtenir la représentation dimensionnelle la plus intéressante sur le plan physique, nous choisirons les grandeurs de référence de la façon suivante :

$$
L=e^{*} \quad V=\omega e^{*}
$$

On définit alors les quantités sans dimensions :

$$
\vec{V}=\frac{\vec{V}^{*}}{V} \quad p=\frac{p^{*}}{\mu \omega} \overrightarrow{O M}=\frac{\overrightarrow{O M}^{*}}{L} z=\frac{z^{*}}{L} r=\frac{r^{*}}{L}
$$

En reportant ces grandeurs dans les équations (1) et les conditions aux limites (2), on obtient les relations adimensionnelles correspondantes :

$$
\left.\begin{array}{l}
\operatorname{Re} \overline{\overline{\operatorname{grad}}} \vec{V} \cdot \vec{V}=-\overrightarrow{\operatorname{grad}} p+\Delta \vec{V} \\
\operatorname{div} V \stackrel{\overrightarrow{=}}{=} 0
\end{array}\right\}
$$

avec les conditions aux limites suivantes :

$$
\left.\begin{array}{l}
\vec{V}(r, \theta, 0)=\overrightarrow{0} \\
\vec{V}(r, \theta, 1)=r \vec{j}
\end{array}\right\}
$$

\subsection{Recherche d'une solution}

Le problème régi par les systèmes (3) et (4) ressemble à un type de problème déjà traité au moyen de solutions exactes de l'équation de Navier-Stockes [9]. Il existe une solution affine du problème, obtenue en posant :

$$
\left.\begin{array}{l}
u=r f^{\prime}(z, R e) \\
v=r g\left(z, R_{e}\right) \\
w=-2 f^{\prime}\left(z, R_{e}\right) \\
p=r^{2} h\left(z, R_{e}\right)+l\left(z, R_{e}\right)
\end{array}\right\}
$$


On remplace vitesse et pression par leurs expressions respectives dans le système (5); pour toute valeur de la coordonnée radiale $r$, on obtient :

$$
\left.\begin{array}{l}
\operatorname{Re}\left(f^{\prime 2}-2 f^{\prime \prime} f-g^{2}\right)=-2 h+f^{\prime \prime \prime} \\
\operatorname{Re}\left(2 f^{\prime} g-2 f g^{\prime}\right)=g^{\prime \prime} \\
h^{\prime}=0 \\
-4 f^{\prime} f=l^{\prime}-2 f^{\prime \prime}
\end{array}\right\}
$$

Les conditions aux limites (4) deviennent :

$$
\left.\begin{array}{rl}
f^{\prime}(0, R e) & =0 \\
z=0 & g, R e)=0 \\
f(0, R e) & =0
\end{array}\right\}
$$

La résolution du système d'équations différentielles (6) et de ses conditions aux limites associées (7) et (8) a été effectuée numériquement en utilisant un programme de calcul mis au point antérieurement [9] et adapté à notre cas [10]. L'intégration numérique, réalisée par la méthode de Newton utilise la technique du tir corrigée. Le calcul pas à pas débute sur la paroi de chacun des disques et une condition de raccordement doit être vérifiée sur une surface fictive. Celle-ci est parallèle au plan des disques et située à la côte $z_{\mathrm{f}}=0,5$. À cet effet, le domaine fluide considéré a été partagé en deux zones repérées par l'indice $i(i=1,2)$ situées de part et d'autre du plan de raccordement. En tenant compte de cette surface de raccordement, on distingue deux conditions indépendantes l'une de l'autre (une sur la vitesse et l'autre liée à la continuité des composantes du tenseur des contraintes) qui vont nous permettre de bien mener la résolution numérique de notre type d'écoulement.

\subsection{Système différentiel}

La méthode d'intégration utilisée, qui impose la division en deux du domaine fluide, conduit donc à la résolution des deux systèmes différentiels :

$$
\begin{aligned}
& R e\left(f_{i}^{\prime 2}-2 f_{i}^{\prime \prime} f_{i}-g_{i}^{2}\right)=-2 h_{i}+f_{i}^{\prime \prime \prime} \\
& R_{\mathrm{e}}\left(2 f_{i}^{\prime} g_{i}-2 f_{i} g_{i}^{\prime}\right)=g_{i}^{\prime \prime} \\
& h_{i}^{\prime}=0 \\
& -4 f^{\prime} f_{i}=l_{i}^{\prime}-2 f^{\prime \prime}
\end{aligned}
$$

avec $i=1,2$

Les conditions aux limites associées, déduites des relations (7) et (8) sont :

$$
\left.z=1 \quad \begin{array}{l}
f_{1}^{\prime}(1, R e)=0 \\
g_{1}(1, R e)=1 \\
f_{1}(1, R e)=0
\end{array}\right\}
$$

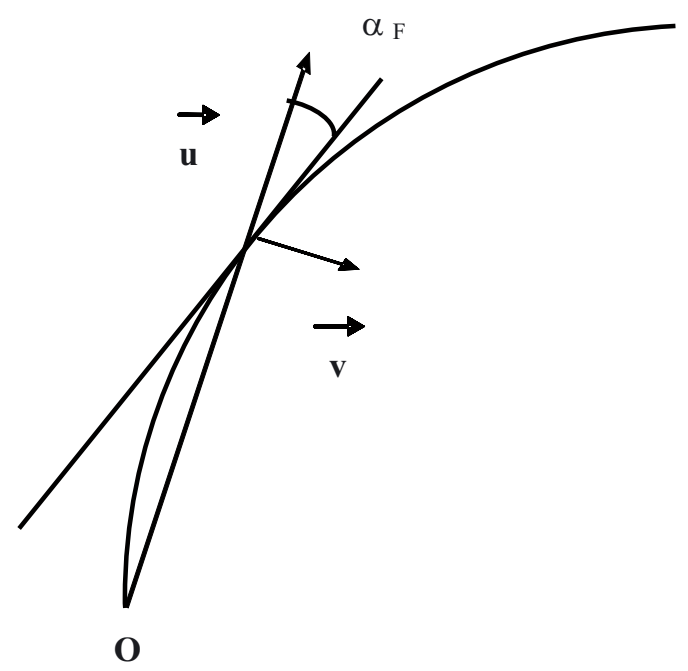

Fig. 2. Ligne de courant pariétal.

$$
\left.z=0 \quad \begin{array}{l}
f_{2}^{\prime}(0, R e)=0 \\
g_{2}(0, R e)=0 \\
f_{2}(0, R e)=0
\end{array}\right\}
$$

\subsection{Lignes de courant pariétales}

Le programme de calcul utilisé a permis d'obtenir les fonctions du système jusqu'à une valeur du nombre de Reynolds de rotation de 270. La direction du vecteur vitesse en un point quelconque de l'écoulement se déduit en fonction des composantes $u$ et $v$. Si on désigne par $\alpha_{\mathrm{F}}$ l'angle formé par ce vecteur vitesse et la direction radiale centrifuge comme le montre la figure $2 ; \alpha_{\mathrm{F}}$ est donné par la relation :

$$
\forall r, \alpha_{\mathrm{F}}(r, z, R e)=\operatorname{Arctg} \frac{v(r, z, R e)}{u(r, z, R e)}=\operatorname{Arctg} \frac{g(z, R e)}{f^{\prime}(z, R e)}
$$

Dans le cas où le repère angulaire est lié au disque tournant à la vitesse angulaire $\omega$, l'angle $\alpha_{\mathrm{T}}$ correspondant est donné par :

$$
\begin{aligned}
\forall r, \alpha_{\mathrm{T}}(r, z, R e) & =\operatorname{Arctg} \frac{v(r, z, R e)-v(r, 1, R e)}{u(r, z, R e)} \\
& =\operatorname{Arctg} \frac{g(z, R e)-g(1, R e)}{f^{\prime}(z, R e)}
\end{aligned}
$$

avec $\forall r, v(r, 1)=1$

Les lignes de courant pariétales à la surface des disques fixe et tournant correspondent à des angles $\alpha_{\mathrm{FP}}$ et $\alpha_{\mathrm{TP}}$ tels que :

$$
\begin{aligned}
& \forall r, \alpha_{\mathrm{FP}}=\lim _{Z \rightarrow 0} \alpha_{\mathrm{F}}(r, z, R e)=\operatorname{Arctg} \frac{g^{\prime}(0, R e)}{f^{\prime \prime}(0, R e)} \\
& \forall r, \alpha_{\mathrm{TP}}=\lim _{Z \rightarrow 1} \alpha_{\mathrm{T}}(r, z, R e)=\operatorname{Arctg} \frac{g^{\prime}(1, R e)}{f^{\prime \prime}(1, R e)}
\end{aligned}
$$


où $z$ et $r$ sont les coordonnées axiale et radiale respectives et $R e$ étant le nombre de Reynolds de rotation tel que :

$$
R e=\frac{\omega e^{* 2}}{v}
$$

où $\omega$ est la vitesse de rotation, $e^{*}$ est l'épaisseur du jeu entre les deux disques et $v$ la viscosité cinématique du fluide utilisé.

Pour étudier un éventuel changement de régime de l'écoulement, il est indispensable de définir un paramètre local. Concernant le disque tournant, le nombre de Reynolds le plus utilisé couramment dans la littérature [11-13] est défini par :

$$
R e_{r}=\frac{\omega r^{* 2}}{v}
$$

Dans notre étude le nombre de Reynolds est basé sur l'épaisseur définie auparavant comme grandeur dimensionnelle de référence $[9,10,14,15]$, par contre on peut désigner par $R e_{r_{0}}$ la valeur prise par $R e_{r}$ à la périphérie des disques, alors $R e_{r_{0}}$ est lié aux deux paramètres adimensionnels $R e$ et $r_{0}$ par la relation :

$$
R e_{r_{0}}=\operatorname{Re} r_{0}^{2}=R e \frac{r_{0}^{* 2}}{e^{* 2}}
$$

La valeur du rayon des deux disques utilisés dans le cadre de nos expériences est : $r_{0}^{*}=83 \mathrm{~mm}$ et il est très supérieur par rapport à l'épaisseur entre les deux disques qui varie entre $2 \mathrm{~mm}$ et $6 \mathrm{~mm}$. En tenant compte de la représentation adimensionnelle la plus intéressante sur le plan physique et du choix des grandeurs de référence, nous avons opté de présenter nos résultats pour un nombre de Reynolds de rotation $R e$ allant jusqu'à 100 pour le disque tournant. Au-delà de cette valeur l'un des paramètres essentiels de notre étude à savoir l'angle des lignes de courant pariétales reste constant [6,8] et le régime de couche limite semble être atteint pour les deux disques $[4,12,16]$.

\section{Resultats}

Les valeurs de $g^{\prime}(0), g^{\prime \prime}(1), f^{\prime \prime}(0)$ et $f^{\prime \prime}(1)$ contenues dans les équations (14) et (15) ont été calculées lors de l'intégration numérique pour les différents nombres de Reynolds considérés [10]. Ces valeurs dépendent du nombre de Reynolds comme le montrent les graphes des figures 3 à 6 .

\subsection{Variation des lignes de courant pariétales en fonction du nombre de Reynolds de rotation}

\subsubsection{Disque tournant}

Sur la figure 7 , on observe que lorsque le nombre de Reynolds augmente $\alpha_{\mathrm{TP}}$ décroît rapidement et ce tant que $R e$ est petit mais à partir de $R e$ égal à 25 , il garde

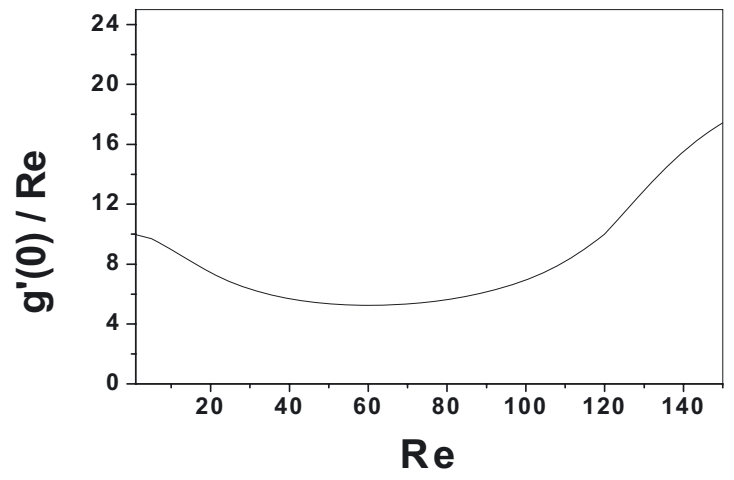

Fig. 3. Variation de la fonction de courant $g^{\prime}(\mathrm{O})$ par rapport au Reynolds de rotation Re.

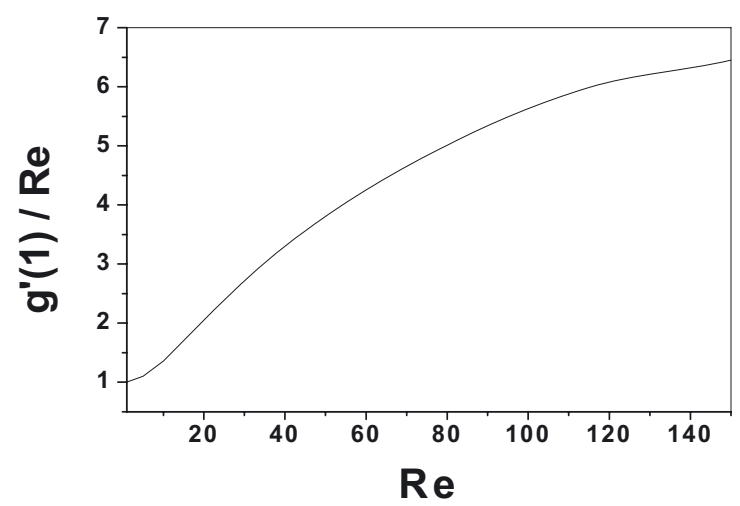

Fig. 4. Variation de la fonction de courant $g^{\prime}(1)$ par rapport au Reynolds de rotation $R e$.

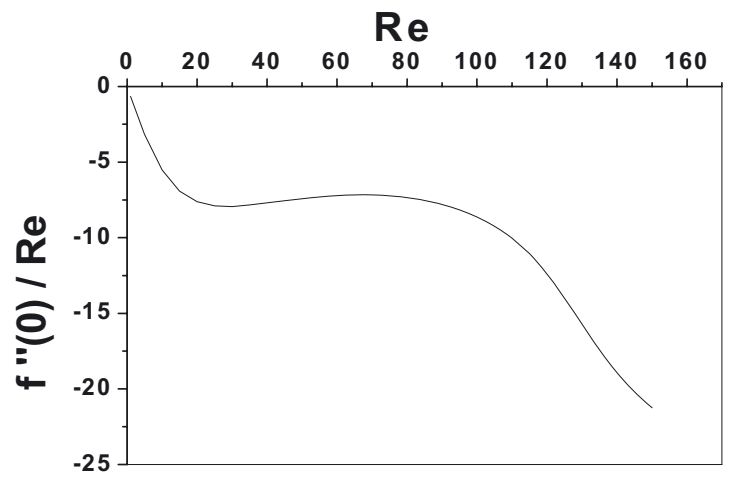

Fig. 5. Variation de la fonction de courant $f^{\prime \prime}(\mathrm{O})$ par rapport au Reynolds de rotation $R e$.

une valeur pratiquement constante voisine de 48 degrés que nous allons tenter d'expliquer par la suite.

Lorsque $R e$ est suffisamment grand pour que le régime de couche limite soit atteint au voisinage de la surface du disque tournant, il est possible de comparer nos résultats avec ceux de Rogers et al. [5] qui ont considéré le cas de la rotation d'un disque de vitesse angulaire $\omega$ au sein d'un fluide tournant en bloc à la vitesse $s \omega$ avec $0<s<1$. En utilisant les valeurs des fonctions $g^{\prime}(s)$ et $f^{\prime \prime}(s)$ qu'ils ont calculé pour différentes valeurs du facteur de rotation $s$, il est possible d'en déduire la valeur de l'angle $\alpha_{\mathrm{TP}}$ 


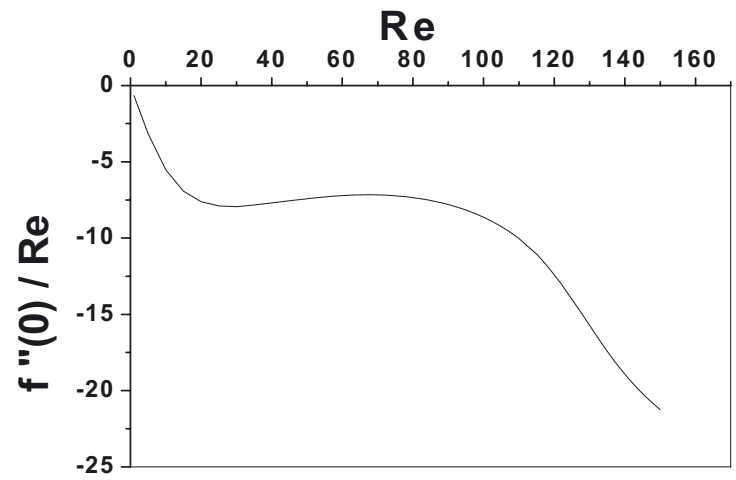

Fig. 6. Variation de la fonction de courant $f^{\prime \prime}(1)$ par rapport au Reynolds de rotation $R e$.

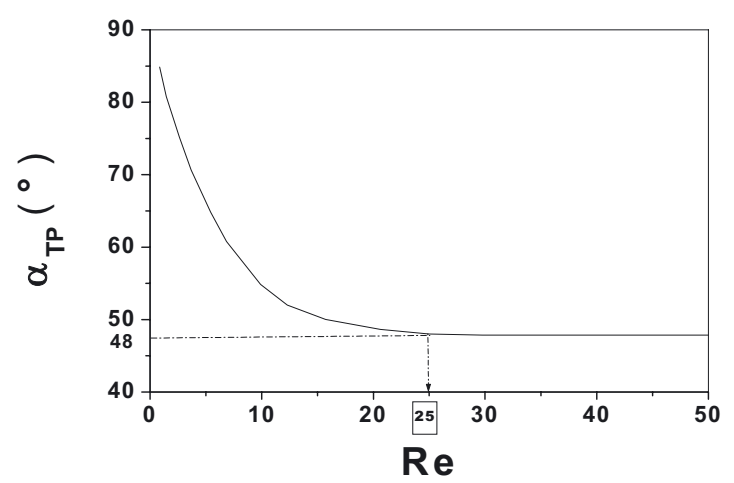

Fig. 7. Variation de l'angle $\alpha_{\mathrm{TP}}$ disque tournant en fonction du Reynolds de rotation Re.

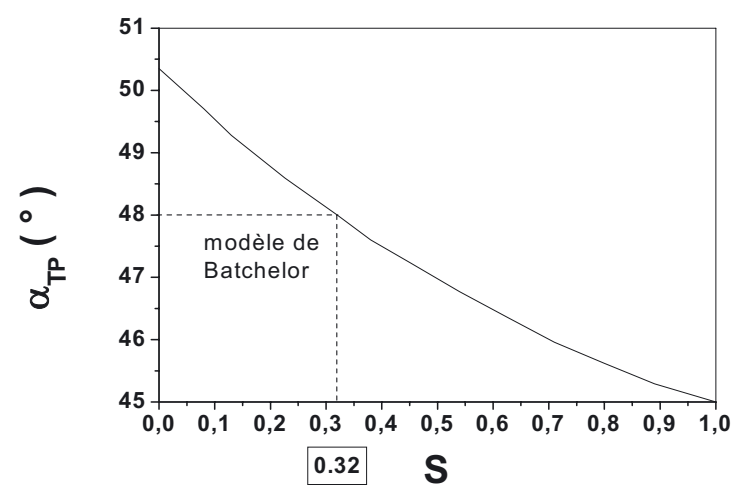

Fig. 8. Variation de l'angle $\alpha_{\mathrm{TP}}$ par rapport au facteur de rotation $s$.

correspondant. La courbe de variation de $\alpha_{\mathrm{TP}}$ en fonction du paramètre $s$ est tracée sur la figure 8

Dans le cas d'un disque tournant face à un disque fixe, les modèles d'écoulement utilisés aux grands nombres de $R e$ prévoient en général l'existence d'un noyau de fluide parfait tournant en bloc à la vitesse $0,3 \omega$ comme le montrent les travaux de Batchelor [4]. La figure 8 permet de constater que la valeur $s=0,32$ correspond à un angle $\alpha_{\mathrm{TP}}$ voisin de 48 degrés, qui n'est autre que la valeur limite de la courbe de la figure 7. L'intégration numérique conduit donc pour les grands nombres de Reynolds à

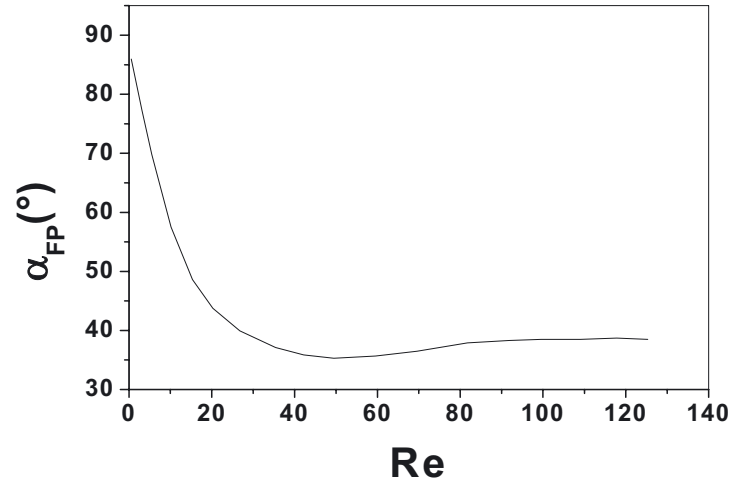

Fig. 9. Variation de l'angle $\alpha_{\mathrm{FP}}$ par rapport au nombre de Reynolds de rotation Re.

un résultat identique à celui obtenu en intégrant les équations de Navier-Stokes à l'aide des hypothèses de la couche limite. La comparaison des deux méthodes montre également que le régime de couche limite semble atteint dès que $R e$ dépasse une valeur voisine de 25 .

\subsubsection{Disque fixe}

La variation de l'angle $\alpha_{\mathrm{FP}}$ en fonction du Re est représentée par la figure 9. Pour les valeurs faibles du $R e, \alpha_{\mathrm{FP}}$ décroît rapidement lorsque le $R e$ augmente, il passe par minimum pour $R e$ égal à 50 avant de croître ensuite lentement avec $R e$ et tendre vers une valeur de 39,30 degrés. Cette dernière coïncide avec celle obtenue par Bodewat [3] dans le cadre des hypothèses de la couche limite. La valeur de $\alpha_{\mathrm{FP}}$ obtenue est alors $39,30^{\circ}$; celle déduite des résultats obtenus à partir des mêmes hypothèses par Rogers et al. [5] est de 39,6 degrés. La comparaison entre ces valeurs limites et celle de la courbe $\alpha_{\mathrm{FP}}$ de la figure 9 permet de constater que le régime de couche limite est atteint à proximité du disque fixe dès que $R e$ dépasse 80 .

\subsection{Lignes de courant pariétales sur le disque tournant}

Un des avantages de la technique de visualisation par dépôt électrolytique est d'obtenir, dans le cas d'un obstacle mobile, des valeurs de $\alpha_{\mathrm{TP}}$ pour l'écoulement relatif, c'est-à-dire par rapport à un repère lié à la paroi en mouvement. L'angle tel qu'il est défini sur la figure 2 est donc caractéristique du mouvement relatif du fluide par rapport au disque tournant à la vitesse angulaire $\omega$.

Le dispositif utilisé lors des essais est schématisé figure 10. L'ensemble disque fixe-disque tournant dont l'écartement et le parallélisme ont été réglés au préalable sont placés à l'intérieur d'une cuve cylindrique de $500 \mathrm{~mm}$ de diamètre et de $300 \mathrm{~mm}$ de hauteur. Ce récipient qui contient la solution électrolytique repose sur un plateau doté d'un mouvement vertical d'amplitude maximum $2540 \mathrm{~mm}$. Ce système de déplacement utilisant un vérin pneumatique il permet de placer les disques au sein 


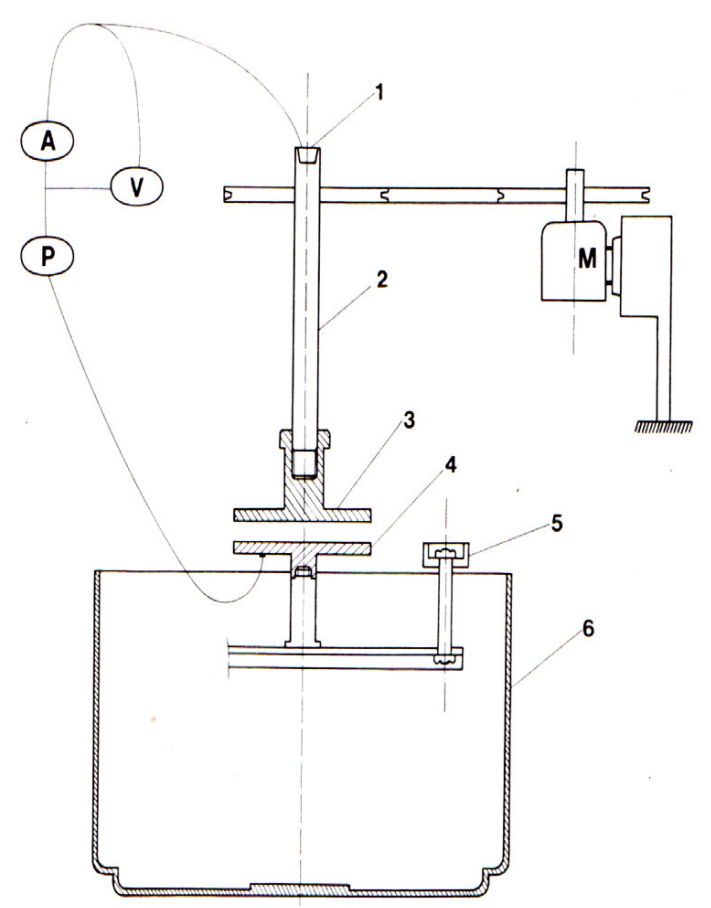

Fig. 10. Montage expérimental, $\mathrm{A}$ : ampèremètre, $\mathrm{M}$ : moteur, $\mathrm{P}$ : potentiomètre, $\mathrm{V}$ : voltmètre, 1 : contact électrique tournant, 2 : arbre tournant, 3 : disque tournant, 4 : disque fixe, 5 : support de disque fixe, 6 : cuve d'électrolyse.

du bain d'électrolyse avant chaque opération de visualisation et de le dégager rapidement à la fin de chaque expérience afin de procéder à l'observation.

Chaque disque a un diamètre de $166 \mathrm{~mm}$ et a été réalisé en deux exemplaires, l'un en cuivre et l'autre en duralumin. Le disque en duralumin est utilisé comme cathode lorsqu'on désire visualiser l'écoulement pariétal sur ce même disque. Le disque de cuivre joue au contraire le rôle d'anode pour obtenir une visualisation sur le disque opposé. Le disque tournant est fixé à l'extrémité d'un arbre vertical. Cet arbre est relié à un bâti par un ensemble de deux paliers munis de roulements à billes qui lui assurent une liberté de rotation. Ce dispositif associé à différents rapports de diamètres des poulies a permis d'obtenir des vitesses de rotation du disque comprises entre 1,1 tr.s ${ }^{-1}$ et 9,10 tr.s ${ }^{-1}$. Le contrôle de cette vitesse est assuré de manière continue lors d'un essai par l'intermédiaire d'une cellule photoélectrique associé à un compte-tours électronique. L'erreur relative ainsi commise sur la mesure de $N$ est inférieure à $0,5 \%$.

Le disque fixe est solidaire d'un système de déplacement micrométrique suivant trois directions orthogonales. L'ensemble est relié à un châssis doté également de vis calantes permettant d'obtenir le parallélisme entre les disques fixe et tournant. Ce réglage étant effectué, le système de déplacement micrométrique permet d'assurer la coïncidence des axes des deux disques et de positionner le disque fixe à la distance désirée du disque tournant. La précision des déplacements est de $10^{-2} \mathrm{~mm}$ mais l'erreur sur la valeur du jeu est évaluée à $0,2 \mathrm{~mm}$.

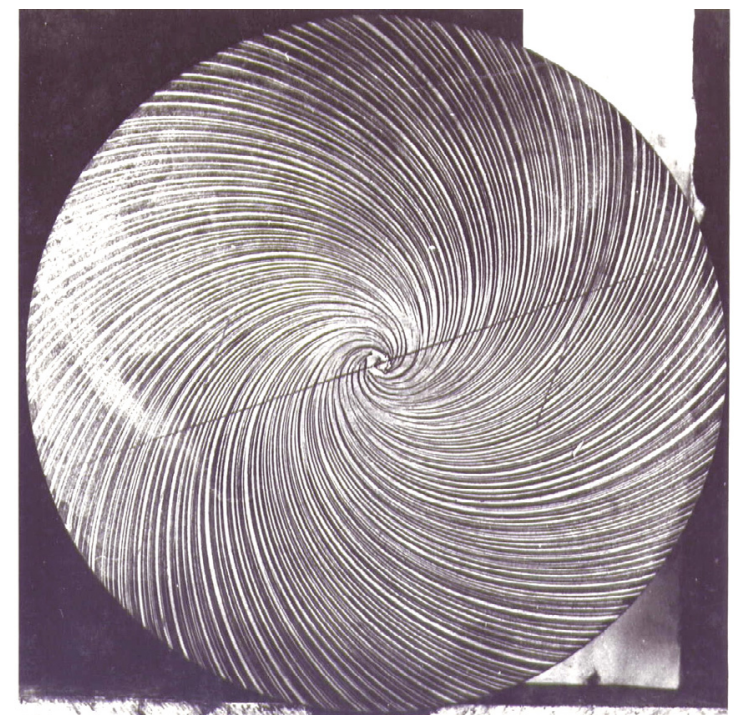

Fig. 11. Lignes de courant pariétales sur un disque tournant pour un nombre de $R e=80$.

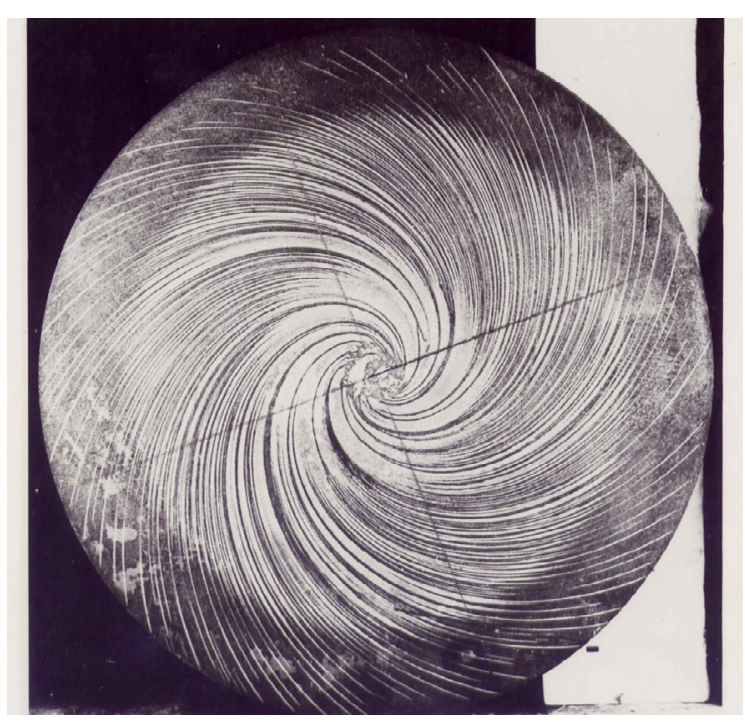

Fig. 12. Lignes de courant pariétales sur un disque tournant pour un nombre de $R e=30$.

Les photographies reproduites sur les figures 11 et 12 obtenues à partir des nos essais expérimentaux illustrent bien les lignes de courant pariétales (appelées souvent dans la littérature spirales logarithmiques) sur le disque tournant. Celles-ci correspondent à deux nombres de Reynolds différents.

\subsubsection{Distribution radiale de l'angle $\alpha_{\text {TP }}$}

Sur la figure 13 ont été portées en fonction du rayon réduit $r=r^{*} / r_{0}^{*}$ les valeurs de l'angle $\alpha_{\mathrm{TP}}$ mesurées sur les photographies obtenues pour quatre valeurs distinctes de l'épaisseur du jeu lorsque la vitesse de rotation est fixée à $9,1 \mathrm{tr} . \mathrm{s}^{-1}$. On constate que $\alpha_{\mathrm{TP}}$ est indépendant de $r$ ainsi que de l'épaisseur réduite $e=e^{*} / r_{0}^{*}$, sauf au voisinage immédiat de la périphérie du disque. 


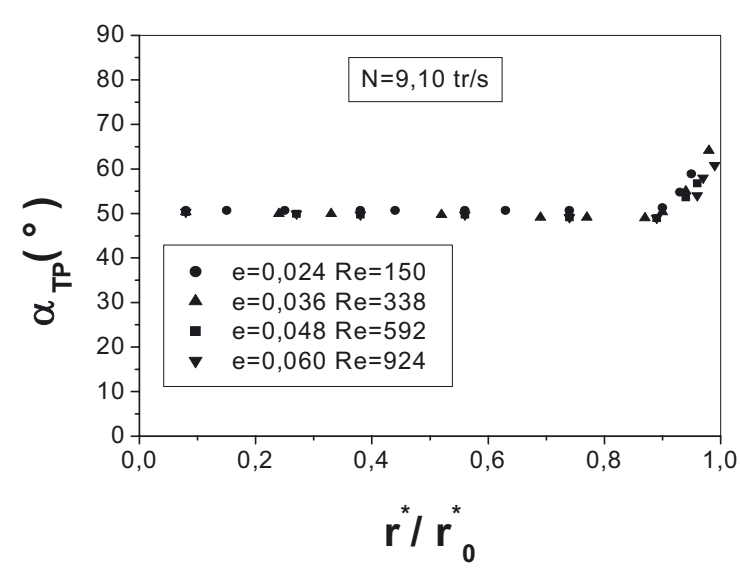

Fig. 13. Évolution de l'angle $\alpha_{\text {TP }}$ en fonction du rayon réduit pour plusieurs valeurs de l'épaisseur $e$ et pour $n=9,10$ tr.s ${ }^{-1}$.

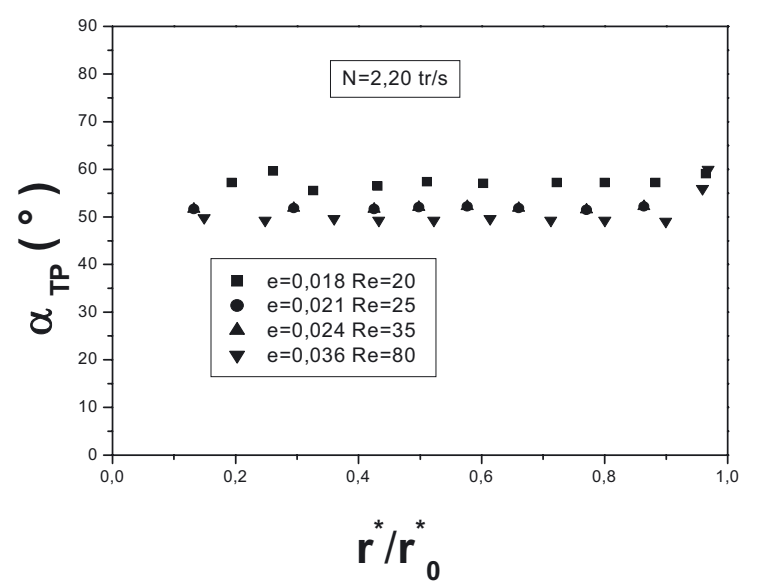

Fig. 14. Évolution de l'angle $\alpha_{\mathrm{TP}}$ en fonction du rayon réduit pour plusieurs valeurs de l'épaisseur $e$ et pour $n=2,20$ tr.s ${ }^{-1}$.

Dans la majorité du domaine situé à proximité du disque tournant, on trouve donc que le champ hydrodynamique est indépendant de l'effet de confinement dû à la présence du disque fixe. Le champ des vitesses est alors conforme à celui prévu théoriquement en supposant le champ de vitesse affine. Il faut cependant noter que la valeur mesurée de $\alpha_{\mathrm{TP}}$ est supérieure d'environ 2 degrés à la valeur calculée à partir des équations du mouvement dans le cadre des hypothèses de type couche limite.

À la périphérie du jeu par contre, dans la région annulaire comprise entre $r=0,9$ et $r=1, \alpha_{\text {TP }}$ est une fonction croissante de $r$. D'autre part, pour une valeur donnée de $r$ telle que $0,9<r<1, \alpha_{\mathrm{TP}}$ augmente quand l'épaisseur du jeu diminue. Ces derniers résultats traduisent l'existence d'un effet d'entrée et de bord dû à la présence du disque fixe.

Les courbes des figures 14 et 15 ont été obtenues à partir d'expériences réalisées avec un disque tournant respectivement à 2,2 tr.s ${ }^{-1}$ et 1,1 tr.s ${ }^{-1}$ pour quatre valeurs distinctes de l'épaisseur du jeu. Comme précédemment on observe que $\alpha_{\mathrm{TP}}$ est indépendant de $r$ sur la majeure partie de la surface du disque tournant puis croît avec le rayon au voisinage de la périphérie.

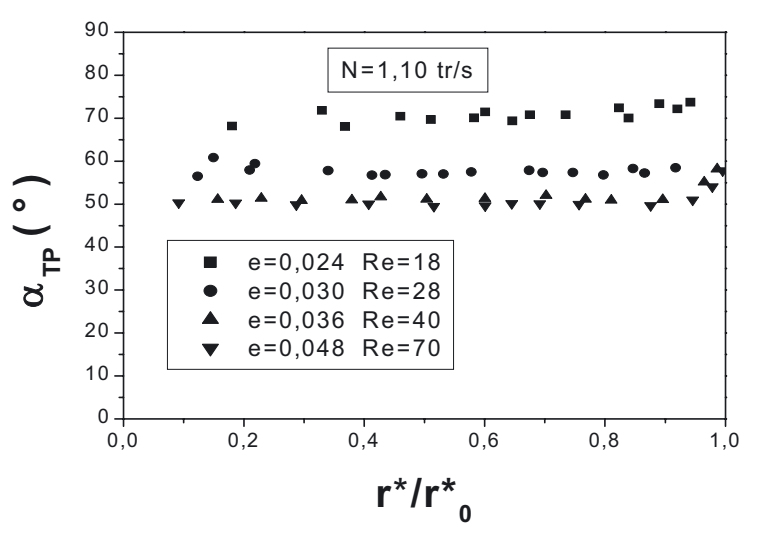

Fig. 15. Évolution de l'angle $\alpha_{\mathrm{TP}}$ en fonction du rayon réduit pour plusieurs valeurs de l'épaisseur $e$ et pour $n=2,20$ tr.s ${ }^{-1}$.

\subsubsection{Effets de bord-Influence du paramètre épaisseur $e$}

La même allure des distributions de l'angle $\alpha_{\mathrm{TP}}(r)$ peut être observée aux faibles vitesses de rotation comme le montre la figure 15. Ces derniers résultats révèlent que l'étendue de la zone annulaire où les effets de bord (les phénomènes d'entrée) se manifestent, progresse en direction de l'axe au fur et à mesure que l'épaisseur du jeu diminue. Ainsi pour une vitesse donnée (caractérisée par la valeur du nombre de $R e_{r_{0}}=\omega r_{0}^{* 2} / v$ ), les effets de bord dus à la dimension finie du rayon du disque s'étendent d'autant plus vers l'intérieur du jeu que le paramètre caractéristique du confinement $e=e^{*} / r_{0}^{*}$ est plus petit.

\subsection{3 Évolution des lignes de courant pariétales en fonction du nombre de Reynolds}

Comme cela peut être constaté sur les figures 13 à 15, l'ordonnée des différents paliers est indépendante du nombre de Reynolds Re lorsque celui-ci est grand. Par contre pour les petites valeurs de ce paramètre, la valeur des lignes de courant pariétales dans la région affine croît lorsque $R e$ diminue ainsi que le prévoient les calculs. Les valeurs de $\alpha_{\mathrm{TP}}$ déduites des paliers correspondant aux diverses expériences réalisées sont portées en fonction de $R e$ sur la figure 16. On constate que la dispersion des points expérimentaux est grande lorsque $R e$ est inférieur à 20. Une explication possible de cette dispersion est l'imprécision affectant la mesure de l'épaisseur du jeu. Celle-ci est de l'ordre de 0,2 mm, soit plus de $10 \%$ en valeur relative lorsque $e^{*}$ est faible, ce qui est le cas aux petits nombres de Reynolds.

Si on compare pour un même nombre de Reynolds les résultats expérimentaux avec théoriques, on constate que les mesures fournissent dans tous les cas une valeur de $\alpha_{\text {TP }}$ supérieure à celle prévue par le calcul.

Pour les valeurs de $R e$ supérieures à 30 , l'écart entre les ordonnées des points expérimentaux et celles de la courbe théorique (qui sont alors indépendantes de $R e$ ) est lui-même constant et égal à 2 degrés. Ceci a été observé dans le cas des expériences réalisées au laboratoire à partir 


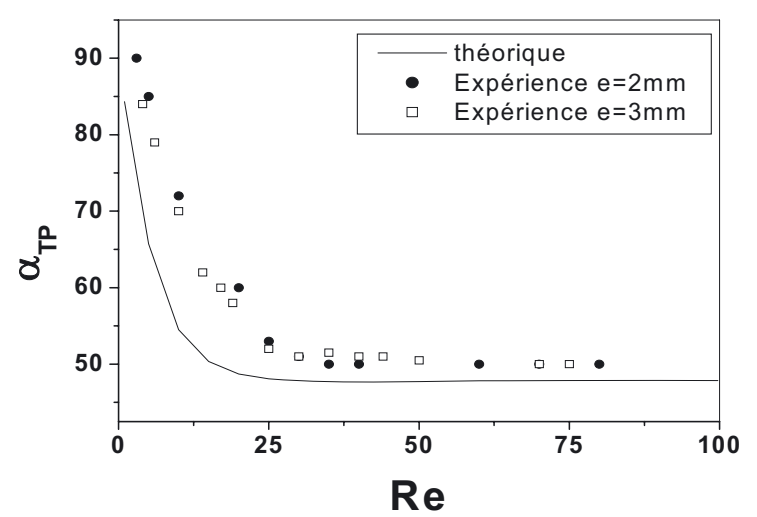

Fig. 16. Évolution de l'angle $\alpha_{\mathrm{TP}}$ en fonction du nombre de Reynolds Re.

d'un disque tournant en milieu non confiné ainsi que lors d'essais analogues rapportés précédemment dans les travaux de Daguenet et Peube [6], Tuhault [7] et Laghouiter et al. [8] ; la valeur de l'angle obtenue par la technique de visualisation par dépôt est légèrement supérieure à celle prévue par le calcul. Cette surélévation s'amplifie lorsque l'expérience est réalisée aux petits nombres de Reynolds et pour $R e=10$, l'écart dépasse 15 degrés.

\section{Conclusions}

L'intégration numérique, réalisée par la méthode de Newton utilise la technique du tir corrigé a permis de montrer que l'écoulement est de type Batchelor, à savoir : l'existence de deux couches limites développées sur chaque disque, séparées par un noyau de fluide tournant à une vitesse de rotation $0,32 \omega$. D'autres chercheurs comme Poncet et al. [12] ont pu obtenir récemment $0,44 \omega$, ce coefficient étant le rapport entre la vitesse tangentielle dans le noyau et celle du disque au même rayon.

La comparaison entre les résultats des calculs théoriques effectués dans le cadre de l'hypothèse d'affinité et les valeurs expérimentales correspondantes montre que la technique de visualisation par dépôt électrolytique donne des résultats satisfaisants aux grands nombres de Reynolds.

Dans une région annulaire périphérique de largeur variable, la valeur de l'angle $\alpha_{\mathrm{TP}}$ (angle des lignes de courant pariétales du disque tournant), au lieu de demeurer constante comme l'exigerait la propriété d'affinité du champ de vitesses dans le cas de disques de rayon infini, augmente avec $r$. Ce fait traduit que l'utilisation de disque de rayon fini entraîne soit une diminution de la vitesse radiale soit une augmentation de la vitesse tangentielle relative du fluide par rapport au disque tournant, soit une combinaison des deux phénomènes. Ainsi on a pu mettre en évidence les effets de bord ou les phénomènes d'entrée liés à la valeur finie du rayon des disques tournant et fixe.

Elle semble par contre moins bien adaptée lorsque le nombre de Reynolds est faible. On observe alors une divergence croissante entre théorie et expérience au fur et à mesure que ce nombre diminue et cela constitue l'un des résultats de nos travaux. Le dépôt électrolytique effectué sur la surface du disque (fixe ou tournant) paraît alors mettre en évidence non pas l'écoulement pariétal mais plutôt celui situé dans une zone de plus en plus éloignée de la paroi.

\section{Références}

[1] T. Von Karman, Uber laminare und turbulente Reibung, Z. Angew. Math. Mech. 1 (1921) 233-252

[2] W.G. Cochran, The flow due to a rotating disc, Proc. Cambridge. Phil. Soc. 30 (1934) 365-375

[3] U.T. Bodewat, Die Drehstrômung uberfesten grunde 7 angew, Math. Mech. 20 (1940) 241-253

[4] G.K. Batchelor, Note on class of solutions of the Navier-Stokes equations representing steady rotationally symétric flow, Quart. J. Mech. Appl. 4 (1951) 29-41

[5] M.H. Rogers, G.N. Lance, The rotationnally symetric flow of a viscous fluid in the presence of an infinite rotating disk, J. Fluid Mech. 7 (1960) 617

[6] M. Daguenet, J.L. Peube, Sur une méthode de visualisation des lignes de courant pariétales, C.R. Acad. de Paris 272 (1971) 351-354

[7] J.L. Tuhault, Mise en ouvre et conditions d'application d'une méthode électrochimique de visualisation des lignes de courant pariétales, Thèse de $3^{\mathrm{e}}$ cycle, Poitiers, France, 1973

[8] F. Laghouiter, A. Daguenet, D. Bodiot, M. Daguenet, Visualisation électrochimique des lignes de mouvement pariétales d'un fluide, Application à la mise en œuvre de la dimicroélectrode, J. Chim. Phys. 72 (1975)

[9] J. Pécheux, J. Contribution à l'étude de l'écoulement entre deux disques produit par débit radial, rotation, Soufflage ou Aspiration, Thèse de Docteur ès Sciences, Poitiers, France, 1976

[10] A. Noureddine, Application de la technique de visualisation par dépôt électrolytique à l'écoulement d'un fluide visqueux entre un disque tournant et un disque fixe, CIMASI 96, 1996, Casablanca, Maroc

[11] S. Poncet, M.-P. Chauve, P. Le Gal, http://www.irphe. univ-mrs.fr/ ${ }^{\sim}$ legal/, Turbulent Rotating Disk Flow with Inward Throughflow, J. Fluid Mech. 522 (2005) 253262

[12] S. Poncet, M.P. Chauve, R. Schiestel, Ecoulements tournants : de Batchelor à Stewartson, $17^{\mathrm{e}}$ Congrés Français de Mécanique, France, 2005

[13] C. Nore, O. Daube, F. Moissy, L. Quartier, M. Tartar, L.S. Tckerman, S. Xin, Instabilités tridimensionnelles de l'écoulement de von Karman entre disques contra-rotatifs, Rencontre du non-linéaire, 2005

[14] A. Noureddine, F.Z. Medjaoui, Mise en évidence des phénomènes d'entrée par la visualisation de l'écoulement pariétal au voisinage du disque tournant, C.A.F.M.C 06, Université de Béjaïa, 2006

[15] L.M. Wistoskowski, I. Delbende, J.S. Walker, P. Le Quéré, Axisymmetric stability of the flow between two exactly counter-rotating disks with large aspect ratio, J. Fluid Mech. 546 (2006) 193-202

[16] K. Stewartson, On flow between two rotating coaxial disks, Proc. Camb. Phil. Soc. 49 (1953) 333-441 